CUBO A Mathematical Journal

Vol.21, Noㅜ, (49-60). April 2019

\title{
Certain integral Transforms of the generalized Lommel-Wright function
}

\author{
S. HAQ \\ Department of Applied Mathematics, Faculty of Engineering and Technology, \\ Aligarh Muslim University, Aligarh-202002, UP, India \\ sirajulhaq0070gmail.com \\ K.S. NISAR \\ Department of Mathematics, College of Arts and Science, \\ Prince Sattam bin Abdulaziz University, Wadi Aldawaser, Riyadh region 11991, Saudi Arabia \\ ksnisar1@gmail.com \\ A.H. KHAN \\ Department of Applied Mathematics, Faculty of Engineering and Technology, \\ Aligarh Muslim University, Aligarh-202002, UP, India \\ ahkhan.amu@gmail.com \\ D.L. SUTHAR \\ Department of Mathematics, Wollo University, \\ Ethiopia \\ dlsuthar@gmail.com
}

\begin{abstract}
The aim of this article is to establish some integral transforms of the generalized Lommel-Wright functions, which are expressed in terms of Wright Hypergeometric function. Some integrals involving trigonometric, generalized Bessel and Struve functions are also indicated as special cases of our main results.
\end{abstract}




\section{RESUMEN}

El objetivo de este artículo es establecer algunas transformadas integrales de las funciones generalizadas de Lommel-Wright, que se expresan en términos de la función hipergeométrica de Wright. Algunas integrales que involucran funciones trigonométricas, de Bessel generalizadas y de Struve también se obtienen como casos especiales de nuestros resultados principales.

Keywords and Phrases: Gamma function, generalized Wright hypergeometric function $\mathrm{p}_{\mathrm{q}}$, generalized Lommel-Wright functions $J_{v, \lambda}^{\mu m}(z)$, Integral Transforms.

2010 AMS Mathematics Subject Classification: 33B20, 33B15, 65R10, 33C20. 


\section{Introduction}

The k-Pochhammer symbol $(\lambda)_{v, k}$ is defined $($ for $v, \lambda \in \mathbb{C} ; k \in \mathbb{R}$ ) by 4 ]

$$
(\lambda)_{v, k}=\frac{\Gamma_{k}(\lambda+v k)}{\Gamma_{k}(\lambda)} \quad(\lambda \in \mathbb{C} / 0)
$$

and the k-gamma function has the relation

$$
\Gamma_{k}(z)=k^{z / k-1} \Gamma(z / k)
$$

is such that $\Gamma_{k}(z) \rightarrow \Gamma(z)$ if $k \rightarrow 1$.

The Wright hypergeometric function defined by the series 21]

$$
p \psi_{q}\left[\begin{array}{c}
\left(\alpha_{1}, A_{1}\right), \ldots,\left(\alpha_{p}, A_{p}\right) ; \\
\left(\beta_{1}, B_{1}\right), \ldots,\left(\beta_{q}, B_{q}\right)
\end{array}\right]=\sum_{k=0}^{\infty} \frac{\prod_{j=1}^{p} \Gamma\left(\alpha_{j}+A_{j} k\right) z^{k}}{\prod_{j=1}^{q} \Gamma\left(\beta_{j}+B_{j} k\right) k !},
$$

where the coefficients $A_{1}, \ldots, A_{p}$ and $B_{1}, \ldots, B_{q}$ are positive real numbers such that

$$
1+\sum_{j=1}^{q} B_{j}-\sum_{j=1}^{p} A_{j} \geq 0 .
$$

can be slightly generalized (1.3) as given below.

$$
p \psi_{q}\left[\begin{array}{c}
\left(\alpha_{1}, 1\right), \ldots,\left(\alpha_{p}, 1\right) ; \\
\left(\beta_{1}, 1\right), \ldots,\left(\beta_{q}, 1\right) ;
\end{array}\right]=\frac{\prod_{j=1}^{p} \Gamma\left(\alpha_{j}\right)}{\prod_{j=1}^{q} \Gamma\left(\beta_{j}\right)}{ }_{p} F_{q}\left[\begin{array}{c}
\alpha_{1}, . ., \alpha_{p} ; \\
\beta_{1}, \ldots, \beta_{q} ;
\end{array}\right],
$$

where ${ }_{p} F_{q}$ is the generalized hypergeometric function defined by [19, 21]

$$
{ }_{p} F_{q}\left[\begin{array}{c}
\alpha_{1}, \ldots, \alpha_{p} ; \\
\beta_{1}, \ldots, \beta_{q}
\end{array}\right]=\sum_{k=0}^{\infty} \frac{\left(\alpha_{1}\right)_{n}, \ldots,\left(\alpha_{p}\right)_{n} z^{n}}{\left(\beta_{1}\right)_{n}, \ldots,\left(\beta_{q}\right)_{n} n !}={ }_{p} F_{q}\left(\alpha_{1}, \ldots, \alpha_{p} ; \beta_{1}, \ldots, \beta_{q} ; z\right),
$$

where $(\lambda)_{n}$ is the well known Pochhammer symbol [21].

The generalization of $(\lambda)_{n}$ is given as

$$
\begin{gathered}
\left.(\lambda)_{n}=\lambda(\lambda+1)(\lambda+2), \ldots,(\lambda+n-1)\right), n>0 \\
(\lambda)_{n}=\prod_{m=1}^{n}(\lambda+m-1), \quad(\lambda)_{0}=1, \lambda \neq 0
\end{gathered}
$$




$$
(\lambda)_{n}=\frac{\Gamma(\lambda+n)}{\Gamma(\lambda)}
$$

Generalized Bessel, Lommel, Struve and Lommel-Wright function have originated from concrete problems in Mechanics, Physics, Engineering and Astronomy.

The series representation of the generalized Lommel Wright function as [8];

$$
\begin{gathered}
\mathrm{J}_{v, \lambda}^{\mu, m}(z)=\sum_{k=0}^{\infty} \frac{(-1)^{\mathrm{k}} \Gamma(\mathrm{k}+1)\left(\frac{z}{2}\right)^{2 \mathrm{k}+v+2 \lambda}}{\Gamma(\lambda+\mathrm{k}+1)^{\mathrm{m}} \Gamma(v+\mathrm{k} \mu+\lambda+1) \mathrm{k} !}, \\
(z \in \mathbb{N} /(-\infty, 0] \mathrm{m} \in \mathbb{N}, \quad v, \lambda \in \mathbb{C}, \mu>0) .
\end{gathered}
$$

Also, we have the following relations of generalized Lommel Wright functions with trigonometric functions and the generalized Bessel function and Struve function:

$$
\begin{aligned}
J_{1 / 2,0}^{1,1}(z) & \left.=\sqrt{(} \frac{2}{\pi z}\right) \sin (z) \\
J_{-1 / 2,0}^{1,1}(z) & \left.=\sqrt{(} \frac{2}{\pi z}\right) \cos (z) \\
J_{v, \lambda}^{\mu, 1}(z) & =\mathbb{J}_{v, \lambda}^{\mu}(z) \\
J_{v, 1 / 2}^{1,1}(z) & =H_{v}(z)
\end{aligned}
$$

Further, we recall the following results [5].

$$
\begin{array}{r}
\int_{0}^{\infty} \mathrm{t}^{\mathrm{u}-1} \exp (-\mathrm{t} / 2) W_{\lambda, \mu}(\mathrm{t}) \mathrm{dt}=\frac{\Gamma(1 / 2+\mu+\mathrm{u}) \Gamma(1 / 2-\mu+\mathrm{u})}{\Gamma(1-\lambda+\mathrm{u})} \\
(\operatorname{Re}(\mathrm{u} \pm \mu)>-1 / 2)
\end{array}
$$

where the Whittaker function $\mathbb{W}_{\lambda, \mu}(t)$ is given in [5, 11].

$$
W_{\lambda, \mu}(t)=\frac{\Gamma(-2 \mu)}{\Gamma(1 / 2-\mu-\lambda)} M_{\lambda, \mu}(t)+\frac{\Gamma(2 \mu)}{\Gamma(1 / 2+\mu-\lambda)} M_{\lambda,-\mu}(t)
$$

where $M_{\lambda, \mu}(t)$ is defined as

$$
M_{\lambda, \mu}(t)=z^{1 / 2+\mu} \exp (-t / 2){ }_{1} F_{1}(1 / 2+\mu+u ; 2 \mu+1 ; t)
$$

\section{Definition 1.1. Euler Transform:}

Let $\rho, \sigma \in \mathbb{C}$ and $\operatorname{Re}(\rho), \operatorname{Re}(\sigma)>0$, then the Euler transform of the function $f(z)$ is defined by

$$
\mathbb{B}(f(z) ; \rho, \sigma)=\int_{0}^{1} z^{\rho-1}(1-z)^{\sigma-1} \mathrm{f}(z) \mathrm{d} z
$$




\section{Definition 1.2. Laplace Transform:}

The Laplace transform of the function $f(t)$ is defined as

$$
\mathrm{F}(\delta)=\mathrm{L}(\mathrm{f}(\mathrm{t}) ; \delta)=\int_{0}^{\infty} \exp (-\mathrm{t} \delta) \mathrm{f}(\mathrm{t}) \mathrm{dt}, \quad \operatorname{Re}(\delta)>0
$$

\section{Definition 1.3. Fourier Transform:}

The following integral gives the Fourier transform

$$
u=\operatorname{Im}[u](w)=\int_{R} u(t) \exp (i w t) d t,
$$

where $\mathfrak{u}=\mathfrak{u}(\mathrm{t})$ be a function of the space $S(R)$ Shwartzian space of the function that decay rapidly at $\infty$ together with all derivatives.

\section{Definition 1.4. The Fractional Fourier Transform (FFT):}

Let $u$ be the function belonging to $\phi(R)$, the Lizorkin space of function, where $\phi(R)=\{\phi \in S(R)\}: \operatorname{Im}[\phi] \in V(R)$ and $V(R)$ is the set of functions defined by $V(R)=\{v \in S(R)\}: V_{0}^{u}=0, n=0,1,2, \ldots$ then FFT of order $\alpha, 0 \leq \alpha \leq 1$ is given by

$$
\mathrm{u}_{\alpha}(w)=\operatorname{Im}_{\alpha}(w)=\int_{R} \exp \left(\mathfrak{i} w^{\alpha} \mathrm{t}\right) \mathfrak{u}(\mathrm{t}) \mathrm{dt}
$$

particularly, if $\alpha=1$ (1.17) reduces to FT and for $w>0$ (1.17) reduces to FFT given by Luchko et al [10].

The aim of this paper is to obtain the Euler, Laplace, Whittaker and Fractional Fourier transforms of Lommel-Wright function.

Various generalizations, integrals, transforms and fractional calculus of special functions have been investigated by many researchers (see, for details, 1, 2, 6, 7, 9, 12, 13, 14, 15, 16, 17, 18, 20,). In this sequel, here, we aim at establishing certain new generalized integral formula involving the

generalized Lommel-Wright function $\mathrm{J}_{v, \lambda}^{\mu, m}(z)$ interesting integral formulas which are derived as special cases.

\section{Main Results}

This section deals with some integral formulas involving Lommel-Wright function. 
Theorem 2.1. For $\mathrm{t} \in \mathbb{N} /(-\infty, 0] \mathrm{m} \in \mathbb{N}, v, \lambda \in \mathbb{C}$ and $\mu>0$, the following integral formula holds true

$$
\begin{aligned}
& \int_{0}^{1} t^{\alpha-1}(1-t)^{\beta-1} J_{\nu, \lambda}^{\mu, m}\left(x t^{\sigma}\right) d t=\left(\frac{x}{2}\right)^{v+2 \lambda} \Gamma(\beta) \\
& \times{ }_{2} \psi_{m+2}\left[\begin{array}{c}
(1,1),(\alpha+v \sigma+2 \lambda \sigma, 2 \sigma) ; \\
(\lambda+1,1), \ldots,(\lambda+1,1),(\nu+\lambda+1, \mu),(\alpha+\beta+v \sigma+2 \lambda \sigma, 2 \sigma) ;
\end{array} \quad-\frac{x^{2}}{4}\right] .
\end{aligned}
$$

Proof. On using (1.8) in the integrand of (2.1) and then interchanging the order of integral sign and summation which is verified by uniform convergence of the involved series under the given conditions we get

$$
\begin{aligned}
& \int_{0}^{1} t^{\alpha-1}(1-t)^{\beta-1} J_{v, \lambda}^{\mu, m}\left(x t^{\sigma}\right) d t \\
& =\left(\frac{x}{2}\right)^{v+2 \lambda} \sum_{k=0}^{\infty} \frac{\Gamma(k+1)\left(-x^{2} / 4\right)^{k}}{\Gamma(\lambda+k+1)^{m} \Gamma(v+k \mu+\lambda+1) k !} \\
& \times \int_{0}^{1} t^{\alpha+\sigma(2 k+v+2 \lambda)-1}(1-t)^{\beta-1} d t .
\end{aligned}
$$

Now using (1.14) in the above equation we get

$$
\begin{aligned}
& \int_{0}^{1} t^{\alpha-1}(1-t)^{\beta-1} J_{v, \lambda}^{\mu, m}\left(x t^{\sigma}\right) d t=\Gamma(\beta)\left(\frac{x}{2}\right)^{v+2 \lambda} \\
& \times \sum_{k=0}^{\infty} \frac{\Gamma(k+1) \Gamma(\alpha+v \sigma+2 \lambda \sigma+2 k \sigma)\left(\frac{-x^{2}}{4}\right)^{k}}{\Gamma(\lambda+k+1)^{m} \Gamma(\alpha+\beta+v \sigma+2 \lambda \sigma+2 k \sigma) \Gamma(v+k \mu+\lambda+1) k !} .
\end{aligned}
$$

Finally, using (1.3) in the above equation, we get our assertion (2.1). This completes the proof of Theorem 2.1.

Theorem 2.2. For $\mathrm{t} \in \mathbb{N} /(-\infty, 0] \mathrm{m} \in \mathbb{N}, \nu, \lambda \in \mathbb{C}$ and $\mu>0$, the following integral formula holds true

$$
\begin{aligned}
& \int_{0}^{\infty} t^{\alpha-1} \exp (-t \delta) J_{v, \lambda}^{\mu, m}\left(x t^{\sigma}\right) d t=\left(\frac{x}{2 \delta^{-\alpha}}\right)^{v+2 \lambda}(\delta)^{-\alpha} \\
& \times{ }_{2} \psi_{m+1}\left[\begin{array}{c}
(1,1),(\alpha+v \sigma+2 \lambda \sigma, 2 \sigma) ; \\
(\lambda+1,1), \ldots,(\lambda+1,1),(\nu+\lambda+1, \mu) ;
\end{array}-\frac{x^{2}}{4 \delta^{2 \sigma}}\right] .
\end{aligned}
$$

Proof. On using (1.8) in the integrand of (2.4) and then interchanging the order of integral sign and summation which is verified by uniform convergence of the involved series under the given 
conditions we get

$$
\begin{aligned}
& \int_{0}^{\infty} t^{\alpha-1} \exp (-\delta t) J_{v, \lambda}^{\mu, m}\left(x t^{\sigma}\right) d t \\
& =\left(\frac{x}{2}\right)^{v+2 \lambda} \sum_{k=0}^{\infty} \frac{\Gamma(k+1)\left(-x^{2} / 4\right)^{k}}{\Gamma(\lambda+k+1)^{m} \Gamma(v+k \mu+\lambda+1) k !} \\
& \times \int_{0}^{\infty} t^{\alpha+\sigma(2 k+v+2 \lambda)-1} \exp (-\delta t) d t .
\end{aligned}
$$

Now using (1.15) in the above equation we get

$$
\begin{aligned}
& \int_{0}^{\infty} t^{\alpha-1} \exp (-\delta t) J_{v, \lambda}^{\mu, m}\left(x t^{\sigma}\right) d t=(\delta)^{-\alpha}\left(\frac{x}{2 \delta^{\sigma}}\right)^{v+2 \lambda} \\
& \times \sum_{k=0}^{\infty} \frac{\Gamma(k+1) \Gamma(\alpha+v \sigma+2 \lambda \sigma+2 k \sigma)\left(\frac{-x^{2}}{4 \delta^{2 \sigma}}\right)^{k}}{\Gamma(\lambda+k+1)^{m} \Gamma(v+k \mu+\lambda+1) k !} .
\end{aligned}
$$

Finally, using (1.3) in the above equation, we get our assertion (2.6). This completes the proof of Theorem 2.2 .

Theorem 2.3. For $\mathrm{t} \in \mathbb{N} /(-\infty, 0] \mathrm{m} \in \mathbb{N}, \nu, \lambda \in \mathbb{C}$ and $\mu>0$, the following integral formula holds true

$$
\begin{aligned}
& \int_{0}^{\infty} t^{\eta-1} \exp (-p t) / 2 W_{\lambda, \mu}(p t) J_{v, \lambda}^{\mu, m}\left(w t^{\delta}\right) d t=\left(\frac{w}{p^{\delta}}\right)^{v+2 \lambda} \\
& \times{ }_{3} \psi_{m+2}\left[\begin{array}{c}
(1,1),(1 / 2+\mu+\eta+\delta v+2 \delta \lambda, 2 \delta),(1 / 2-\mu+\eta+\delta v+2 \delta \lambda, 2 \delta) ; \\
(\lambda+1,1), \ldots,(\lambda+1,1),(\nu+\lambda+1, \mu),(1-\lambda+\eta+v \delta+2 \delta \lambda, 2 \delta) ;
\end{array}-\frac{w^{2}}{4 p^{2 \delta}}\right] .
\end{aligned}
$$

Proof. On using (1.8) in the integrand of (2.7) and then interchanging the order of integral sign and summation which is verified by uniform convergence of the involved series under the given conditions we get

$$
\begin{aligned}
& \int_{0}^{\infty}(\mathfrak{u} / \mathrm{p})^{\eta-1} \exp (-\mathfrak{u} / 2) W_{\lambda, \mu}(u) J_{v, \lambda}^{\mu, m}\left(w(u / p)^{\delta}\right) d u \\
& =\left(\frac{w}{p^{\delta}}\right)^{v+2 \lambda} \sum_{k=0}^{\infty} \frac{\Gamma(k+1)\left(-w^{2} / 4 p^{2 \delta}\right)^{k}}{\Gamma(\lambda+k+1)^{m} \Gamma(v+k \mu+\lambda+1) k !} \\
& \times \int_{0}^{\infty} u^{\eta+\delta(2 k+v+2 \lambda)-1} \exp (-\mathfrak{u} / 2) W_{\lambda, \mu}(u) d u .
\end{aligned}
$$

Now using (1.13) in the above equation we get

$$
\begin{aligned}
& \int_{0}^{\infty} t^{\eta-1} \exp (-p t) / 2 W_{\lambda, \mu}(p t) J_{v, \lambda}^{\mu, m}\left(w t^{\delta}\right) d t=\left(\frac{w}{p^{\delta}}\right)^{v+2 \lambda} \\
& \times \sum_{k=0}^{\infty} \frac{\Gamma(k+1) \Gamma(1 / 2+\mu+\eta+2 k \delta+\delta v+2 \delta \lambda) \Gamma(1 / 2-\mu+\eta+2 k \delta+\delta v+2 \delta \lambda)\left(\frac{-w^{2}}{4 p^{2 \delta}}\right)^{k}}{\Gamma(\lambda+k+1)^{m} \Gamma(v+k \mu+\lambda+1) \Gamma(1-\lambda+\eta+2 k \delta+\delta v+2 \delta \lambda) k !} .
\end{aligned}
$$


Finally, using (1.3) in the above equation, we get our assertion (2.9). This completes the proof of Theorem 2.3 .

\section{Special Cases}

In this section, we get some integral formulas involving trigonometric function and generalized Lommel-Wright function as follows:

Corollary 3.1. If we take $\mathrm{m}=1, \mu=1, \lambda=0$ and $\nu=1 / 2$ in (2.1) and then by using (1.9), we derive the following integral formula:

$$
=\sqrt{\pi}\left(\frac{x}{2}\right) \Gamma(\beta){ }_{1} \psi_{2}\left[\begin{array}{cr}
\int_{0}^{1} t^{\alpha-\sigma / 2-1}(1-t)^{(\beta-1)} \sin \left(x t^{\sigma}\right) d t \\
(\alpha+\sigma / 2,2 \sigma) ; & -\frac{x^{2}}{4} \\
(3 / 2,1),(\alpha+\beta+\sigma / 2,2 \sigma) ; &
\end{array}\right]
$$

Corollary 3.2. If we take $\mathrm{m}=1, \mu=1, \lambda=0$ and $\nu=1 / 2$ in (2.4) and then by using (1.9), we derive the following integral formula:

$$
\begin{array}{r}
\int_{0}^{\infty} t^{\alpha-\sigma / 2-1} \exp (-\delta \mathrm{t}) \sin \left(x \mathrm{t}^{\sigma}\right) \mathrm{dt} \\
=\delta^{-\alpha} \sqrt{\frac{\pi}{\delta^{\sigma}}}\left(\frac{x}{2}\right) \Gamma(\beta){ }_{1} \psi_{1}\left[\begin{array}{cc}
(\alpha+\sigma / 2,2 \sigma) ; & \\
(3 / 2,1) ; & -\frac{x^{2}}{4 \delta^{2 \sigma}}
\end{array}\right]
\end{array}
$$

Corollary 3.3. Further if we take $\mathrm{m}=1, \mu=1, \lambda=0$ and $v=1 / 2$ in (2.7) and then by using (1.9), we derive the following integral formula:

$$
=w \sqrt{\frac{\pi}{2 p^{\delta}}} 2 \psi_{2}\left[\begin{array}{cc}
\int_{0}^{\infty} t^{\eta-\delta / 2-1} \exp (-p t / 2) W_{\lambda, \mu}(p t) \sin \left(w t^{\delta}\right) d t \\
(3 / 2,1),(\eta+\delta / 2+1,2 \delta) ; & -\frac{w^{2}}{4 p^{2 \delta}}
\end{array}\right]
$$

Corollary 3.4. If we take $m=1, \mu=1, \lambda=0$ and $v=-1 / 2$ in (2.1) and then by using (1.10), we derive the following integral formula:

$$
=\sqrt{\pi} \Gamma(\beta){ }_{1} \psi_{2}\left[\begin{array}{cr}
\int_{0}^{1} t^{\alpha-\sigma / 2-1}(1-t)^{(\beta-1)} \cos \left(\alpha t^{\sigma}\right) d t \\
(\alpha-\sigma / 2,2 \sigma) ; & -\frac{x^{2}}{4} \\
(1 / 2,1),(\alpha+\beta-\sigma / 2,2 \sigma) ; &
\end{array}\right]
$$


Corollary 3.5. If we take $\mathrm{m}=1, \mu=1, \lambda=0$ and $v=-1 / 2$ in (2.4) and then by using (1.10), we derive the following integral formula:

$$
\begin{aligned}
& \int_{0}^{\infty} t^{\alpha-\sigma / 2-1} \exp (-\delta t) \cos \left(x t^{\sigma}\right) d t \\
& =\delta^{(\sigma-\alpha)} \sqrt{\pi}_{1} \psi_{1}\left[\begin{array}{cc}
(\alpha-\sigma / 2,2 \sigma) ; & \\
(1 / 2,1) ; & -\frac{\chi^{2}}{4 \delta^{2 \sigma}}
\end{array}\right]
\end{aligned}
$$

Corollary 3.6. Further if we take $\mathrm{m}=1, \mu=1, \lambda=0$ and $v=-1 / 2$ in (2.7) and then by using (1.10), we derive the following integral formula:

$$
=w \sqrt{\frac{\pi}{2}} 2 \psi_{2}\left[\begin{array}{cc}
\int_{0}^{\infty} t^{\eta-\delta / 2-1} \exp (-p t / 2) W_{\lambda, \mu}(p t) \cos \left(w t^{\delta}\right) d t \\
(1 / 2,1),(\eta-\delta / 2+1,2 \delta) ; & -\frac{w^{2}}{4 p^{2 \delta}}
\end{array}\right]
$$

Corollary 3.7. If we take $\mathrm{m}=1$ in (2.1) and then by using (1.11), we derive the following integral formula:

$$
\times_{2} \psi_{3}\left[\begin{array}{cr}
\int_{0}^{1} t^{\alpha-1}(1-t)^{(\beta-1)} \mathbb{J}_{\nu, \lambda}^{\mu}\left(x t^{\sigma}\right) d t=\left(\frac{x}{2}\right)^{v+2 \lambda} \Gamma(\beta) \\
(1,1),(\alpha+v \sigma+2 \lambda \sigma, 2 \sigma) ; & -\frac{x^{2}}{4} \\
(\lambda+1,1),(\nu+\lambda+1, \mu),(\alpha+\beta+v \sigma+2 \lambda \sigma, 2 \sigma) ; &
\end{array}\right]
$$

Corollary 3.8. If we take $\mathrm{m}=1$ in (2.4) and then by using (1.11), we derive the following integral formula:

$$
=\left(\frac{x}{2}\right)^{v+2 \lambda} \delta^{-\alpha}{ }_{2} \psi_{2}\left[\begin{array}{lc}
(1,1),(\alpha+v \sigma+2 \lambda \sigma, 2 \sigma) ; & \\
(\lambda+1,1),(\nu+\lambda+1, \mu) ; & -\frac{x^{2}}{4 \delta^{2 \sigma}}
\end{array}\right]
$$

Corollary 3.9. Further if we take $\mathrm{m}=1$ in (2.7) and then by using (1.11), we derive the following integral formula:

$$
\times_{3} \psi_{3}\left[\begin{array}{cc}
\int_{0}^{\infty} t^{\eta-1} \exp (-p t / 2) W_{\lambda, \mu}(p t) J_{v, \lambda}^{\mu}\left(w t^{\delta}\right) d t=\left(\frac{w}{p^{\delta}}\right)^{v+2 \lambda} \\
(\lambda+1,1),(\nu+\lambda+1, \mu),(1-\lambda+\eta+\delta v+2 \delta \lambda, 2 \delta) ; & -\frac{w^{2}}{4 p^{2 \delta}}
\end{array}\right]
$$


Corollary 3.10. If we take $\mu=1, \mathrm{~m}=1$ and $\lambda=1 / 2$ in (2.1) and then by using (1.12), we derive the following integral formula:

$$
\times_{2} \psi_{3}\left[\begin{array}{cr}
\int_{0}^{1} t^{\alpha-1}(1-t)^{(\beta-1)} \mathbb{H}_{v}\left(x t^{\sigma}\right) d t=\left(\frac{x}{2}\right)^{v+1} \Gamma(\beta) \\
(1,1),(\alpha+v \sigma+\sigma, 2 \sigma) ; & -\frac{x^{2}}{4} \\
(3 / 2,1),(\nu+3 / 2,1),(\alpha+\beta+v \sigma+\sigma, 2 \sigma) ; &
\end{array}\right]
$$

Corollary 3.11. If we take $\mu=1, \mathrm{~m}=1$ and $\lambda=1 / 2$ in (2.4) and then by using (1.12), we derive the following integral formula:

$$
\begin{array}{r}
\int_{0}^{\infty} t^{\alpha-1} \exp (-\delta t) \mathbb{H}_{v}\left(x t^{\sigma}\right) d t=\left(\frac{x}{2 \delta^{\sigma}}\right)^{v+1} \delta^{-\alpha} \\
\times{ }_{2} \psi_{2}\left[\begin{array}{cc}
(1,1),(\alpha+v \sigma+\sigma, 2 \sigma) ; & -\frac{x^{2}}{4 \delta^{2 \sigma}} \\
(3 / 2,1),(v+3 / 2,1) ; &
\end{array}\right]
\end{array}
$$

Corollary 3.12. Further if we take $\mu=1, m=1$ and $\lambda=1 / 2$ in (2.7) and then by using (1.12), we derive the following integral formula:

$$
\times_{3} \psi_{3}\left[\begin{array}{cc}
\int_{0}^{\infty} t^{\eta-1} \exp (-p t / 2) W_{\lambda, \mu}(p t) \mathbb{H}_{v}\left(w t^{\delta}\right) d t=\left(\frac{w}{p^{\delta}}\right)^{v+1} \\
(3 / 2,1),(\eta+3 / 2,1),(\eta+\delta \nu+\delta+1 / 2,2 \delta) ; & -\frac{w^{2}}{4 p^{2 \delta}}
\end{array}\right]
$$




\section{References}

[1] J. Choi and P. Agarwal, Certain unified integrals associated with Bessel functions, Bound. Value Probl., 95, (2013), pages 9.

[2] J. Choi, P. Agarwal, S. Mathur and S.D. Purohit, Certain new integral formulas involving the generalized Bessel function, Bull. Korean Math. Soc., 4, (2014), 995-1003.

[3] J. Choi, K.S. Nisar, Certain families of integral formulas involving Struve function, Bol. Soc. Parana. Mat., 37(3), (2019), 27-35.

[4] R. Díaz and E. Pariguan, On hypergeometric functions and k-Pochhammer symbol, Divulg. Mat., 15, (2007), 179-192.

[5] A. Erdélyi,W. Magnus,F. Oberhettinger and F.G. Tricomi, Tables of Integral Transforms, Vol.2, McGraw-Hill, New York-Toronto-London (1954).

[6] K.S. Gehlot, and J.C. Prajapati, Fractional Calculus of generalized k-wright function, Journal of Fractional Calculus and Applications, 4, (2013), 283-289.

[7] K.S. Gehlot and S.D. Purohit, Fractional Calculus of K-Bessels function , Acta Universitatis Apulensis., 38, (2014), 273-278.

[8] K.B. Kachhia and J.C. Prajapati, On generalized fractional kinetic equations involving generalized Lommel-Wright functions, Alexandria Engineering Journal (elsevier) 55, (2016), 2953-2957.

[9] J.P. Konovska, Theorems on the convergence of series in generalized Lommel-Wright functions. Fract. Calc. Appl. Anal., 10(1),(2007), 59-74.

[10] Y. Luchko, H. Martinez and J. Trujillo, Fractional Fourier transform and some of its applications, Fract. Calc. Appl. Anal., 11, (2008), ,457-470.

[11] A.M. Mathai, R.K. Saxena and H.J. Haubold, The H-function, Theory and Applications, Springer, New York (2010).

[12] K.S. Nisar, D. Baleanu and M.M. Al Qurashi, Fractional calculus and application of generalized Struve function, Springer Plus (2016)5:910,DOI 10.1186/s40064-016-2560-3.

[13] K.S. Nisar, G. Rahman, A. Ghaffar, S.A. Mubeen, new class of integrals involving extended Mittag-Leffler function, J. Fract. Calc. Appl., 9 (1), (2018), 222-231.

[14] S.R. Mondal, K.S. Nisar, Certain unified integral formulas involving the generalized modified k-Bessel function of first kind, Commun. Korean Math. Soc., 32(1), (2017), 4753. 
[15] K.S. Nisar, W.A. Khan, Beta type integral operator associated with Wright generalized Bessel function, Acta Math. Univ. Comenian. (N.S.) 87(1), 117-125(2018).

[16] G. Rahman, A. Ghaffar, K.S. Nisar, S. Mubben, A new class of integrals involving extended Mittag-Leffler function Journal of Fractional Calculus and Applications, 9(1), (2018), 222-231.

[17] K.S. Nisar, W.A. Khan and A.H. Abusufian, Certain Integral transforms of k-Bessel function, Palest. J. Math., 7(1), (2018), 161-166.

[18] K.S. Nisar, D.L. Suthar, S.D. Purohit, M. Aldhaifallah, Some unified integral associated with the generalized Struve function, Proc. Jangjeon Math. Soc.,20(2), (2017), 261-267.

[19] E.D. Rainville, Special Functions, Macmillan, New York, 1960.

[20] A.K. Rathie, A new generalization of generalized hypergeometric function, Matematiche (Catania), 52(2), (1997), 297-310.

[21] H.M. Srivastava, and H.L. Manocha, A treatise on generating functions, John Wily and Sons (Halsted Press, New York,Ellis Horwood, Chichester), 1984. 